\title{
Topical dorzolamide treatment of macular cysts in the enhanced S-cone syndrome patient
}

\author{
Marta Kiszkielis • Wojciech Lubiński • \\ Krzysztof Penkala
}

Received: 6 August 2012/Accepted: 21 December 2012/Published online: 6 January 2013

(C) The Author(s) 2013. This article is published with open access at Springerlink.com

\begin{abstract}
Purpose The purpose of the study was to evaluate the efficacy of a topical form of a carbonic anhydrase inhibitor (dorzolamide) on the foveal function and thickness in the eye of a patient with enhanced S-cone syndrome (ESCS) associated with macular cysts.

Methods Twenty-eight-year-old Polish man with ESCS and macular cysts appearance in the right eye was treated 3 times daily with $2.0 \%$ dorzolamide drops for the period time equal to 6 months. Monthly controls included: best corrected distance visual acuity (BCDVA-logMAR), foveal thickness (optical coherence tomography, OCT) and foveal function (multifocal electroretinography, mfERG).

Results Before treatment, BCDVA in the right eye was equal to $0.26 \log \mathrm{MAR}$, improved to $0.1 \log \mathrm{MAR}$ during the first 3 months and remained stable for the next 3 months. After 6 months, foveal thickness decreased (from 482 to $224 \mu \mathrm{m}$ ) and foveal function improved (the amplitude of P1-wave density increased from 34.8 to $107.3 \mathrm{nV} / \mathrm{deg}^{2}$ ) and was between the
\end{abstract}

M. Kiszkielis $(\bowtie) \cdot$ W. Lubiński

Chair and Clinic of Ophthalmology, Pomeranian Medical

University, Powstańców Wlkp. Street 72, 70-111

Szczecin, Poland

e-mail: martakiszkielis@gmail.com

K. Penkala

Department of Systems, Signals and Electronics

Engineering, West Pomeranian University of Technology,

Szczecin, Poland ranges of normal values. Implicit time of P1-wave remained prolonged.

Conclusions The results of our short-term study suggest potential efficacy of topical dorzolamide treatment in ESCS patients with macular cysts.

Keywords Macular cysts - Enhanced S-cone syndrome Foveal function and thickness - Topical dorzolamide

\section{Introduction}

Enhanced S-cone syndrome (ESCS) first described in 1990 by Marmor et al. [1] is a rare, slowly progressive autosomal recessive inherited retinal degenerative disorder. In most cases of ESCS, NR2E3 gene mutation is responsible for the disease development and leads to abnormal cones and rods differentiation [2]. Characteristic fundus changes usually progress from subtle pigmentary changes with white dots in the early stage to a more severe nummular pigmentary deposition around the vascular arcades accompanied by yellowish dots later on. Cystic macular changes occur frequently, in about 50-75\% of cases [3, 4].

Enhanced S-cone syndrome is diagnosed on the basis of characteristic clinical fundus changes and pathognomonic features of the full-field electroretinography (ERG). There were performed according to the International Society for Clinical Electrophysiology of Vision (ISCEV) standards such as the absence 
of rod response, morphologically similar waveforms of scotopic and photopic responses to the standard stimulus flashes (3.0 ERG) and flicker reduction [4, 5]. The S-cone ERG recording can be performed to confirm the pathophysiologic origin of the disease (the S-cone hyperactivity) and is characterized by abnormally large and delayed waveforms (relative to those with standard stimulation) [3].

Retinal pigment epithelium (RPE) function is abnormal in ESCS retina [6], resulting in macular cysts development. Dorzolamide (topical form of carbonic anhydrase inhibitor) may improve RPE metabolism by activating the pumping function and consequently, it reduces intraretinal cysts and RPE remains adherent to the retina $[7,8]$.

Only 3 case report studies [9-11] described efficacy of topical (2.0\% dorzolamide) and/or oral (acetazolamide) carbonic anhydrase inhibitor in the treatment of macular cysts associated with ESCS. In these 3 patients, increase in visual acuity associated with normalization of foveal microanatomy (illustrated by OCT) was observed. Treatment time in these studies varied from $4[9,10]$ up to 27 months [11]. During the follow-up, visual acuity, foveal thickness and structure in each patient improved and remained stable. These promising initial results were the reason to implement dorzolamide therapy in a newly diagnosed ESCS patient presented in this report (Table 1).

\section{Case}

A twenty-eight-year-old white male was sent to the ophthalmology clinic with the suspicion of ESCS and had following complaints: night vision problems since the childhood and decreased visual acuity since 2 years in the left eye and most recently in the right eye. Informed consent was obtained from the patient before the examination.

Initially, the patient's BCDVA was equal to 0.26 in the right eye and 0.2 in the left eye on a logMAR scale

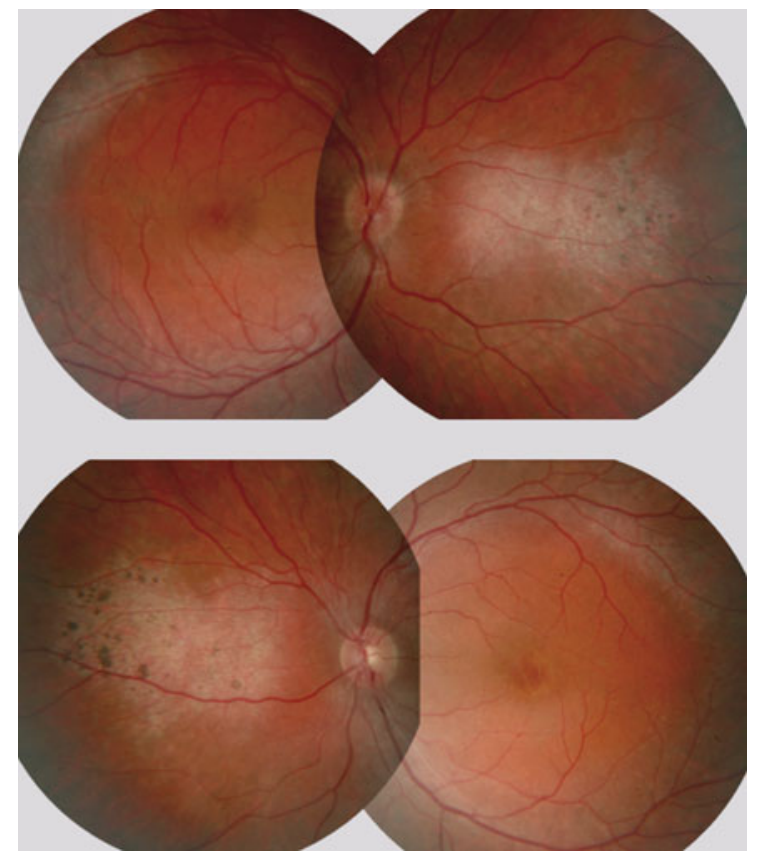

Fig. 1 Fundus photographs, the right and left eye of the 28-year-old man with ESCS

(early treatment diabetic retinopathy study chart). Color vision (Farnsworth Panel-15 D) was normal in both eyes. Fundus exam revealed white dots around the vascular arcades accompanied by single black flecks. Optic disc and vessels were normal. Clinical features of macular cysts were noticed in both eyes (Fig. 1). Macular cysts appearance was confirmed by the OCT test (Zeiss Stratus OCT, Humphrey Instruments model 3000, Carl Zeiss Inc, Dublin, CA, 2007). The cystoid spaces appeared as hyporeflective spaces, interspersed with high-signal septae which represented bridging retinal elements in both eyes. Loss of the normal foveal depression was observed in the right eye (Fig. 2). In the left eye, foveal contour became even convex and dome shaped, as previously described in the literature [6]. Fluorescein angiogram showed absence of any detectable leakage of the dye in the macular area in either eye.

Table 1 .

\begin{tabular}{|c|c|c|c|c|c|c|c|}
\hline & \multirow[t]{2}{*}{ VA logMAR ETDRS } & \multicolumn{2}{|l|}{$\mathrm{FT}(\mu \mathrm{m})$} & \multicolumn{2}{|c|}{ P1 density $\left(\mathrm{nV} / \mathrm{deg}^{2}\right)$} & \multicolumn{2}{|c|}{ Implicit time (ms) } \\
\hline & & $\mathrm{M} \pm \mathrm{SD}$ & Range & Med & Range & $\mathrm{M} \pm \mathrm{SD}$ & Range \\
\hline Normal values & 0.0 & $212 \pm 20$ & $192-232$ & 134.5 & $106.8-148.2$ & $39.2 \pm 2.1$ & $35-43.3$ \\
\hline
\end{tabular}

$M$ mean, $S D$ standard deviation, $M e d$ median 


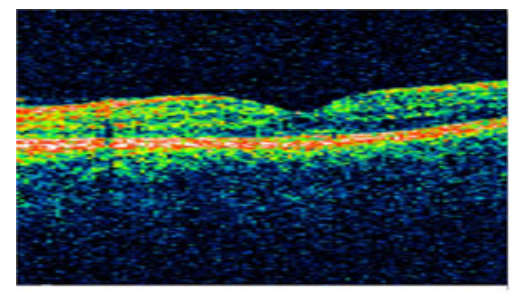

Normal

Trace Array

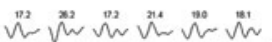

"艹

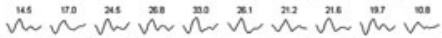

m.

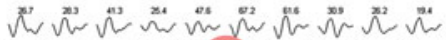

ฉ"

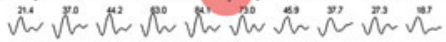

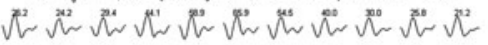

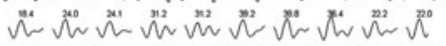

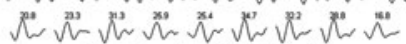

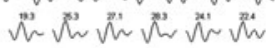

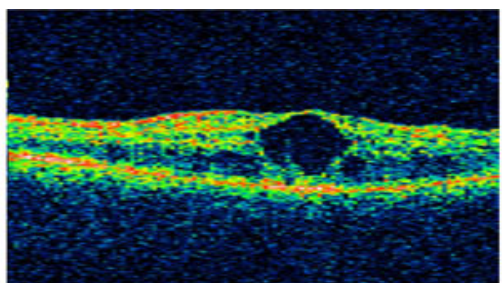

Initially

Trace Array

น

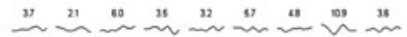

*

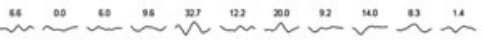

"

"

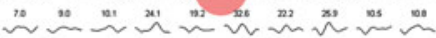

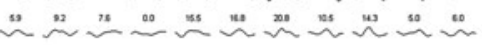

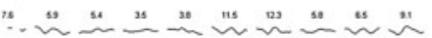

24 $\sim 28$

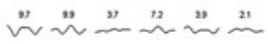

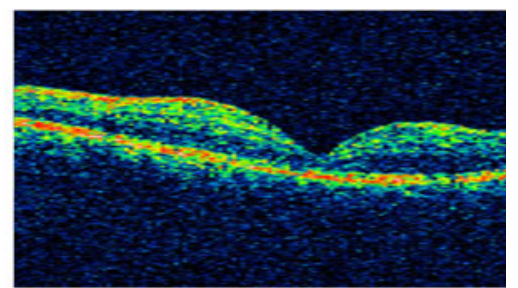

After 6-months Trace Array

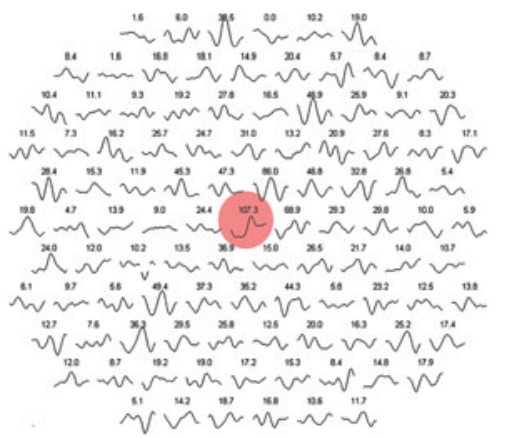

Fig. 2 Optical coherence tomography (OCT) images (above) and mfERG recordings (below) of the right eye of the 28-year-old man with ESCS before and after a 6-month treatment with topical dorzolamide in comparison with the age-matched healthy control

The ERG (ISCEV) [12] was recorded, using Burian-Allen bipolar electrodes (UTAS-E2000, LKC, USA). It showed the absence of rod response, reduced and delayed waveforms in response to white flash $(0 \mathrm{~dB})$, both in scotopic and photopic conditions, reduced and delayed flicker recording (Fig. 3). In the S-cone ERG recordings, blue stimulus $(455 \mathrm{~nm}, 5 \mathrm{~ms}$, $80 \mathrm{~cd} / \mathrm{m}^{2}, 16 \mathrm{~cd} \mathrm{~s} / \mathrm{m}^{2}$ ) was used in the orange background $\left(590 \mathrm{~nm}, 560 \mathrm{~cd} / \mathrm{m}^{2}\right.$ ). Parameters were similar to those performed by Audo et al. [3]. The recordings presented hyper-normal and delayed a- as well as b waves. In mfERG (ISCEV) [13] (RetiScan system, Roland Consult, Germany, 2009), delayed and reduced P1-wave amplitudes were observed in all six analyzed concentric rings. NR2E3 gene sequencing was not performed, because it is not necessary for ESCS diagnosis.

The patient was treated with topical form of $2.0 \%$ dorzolamide 3 times daily for 6 months. Initially, it was applied to both eyes. Left eye treatment was discontinued after 1 month because of inefficacy and cystoid macular changes progression. The probable reason of the left eye treatment failure was prolonged macular cysts appearance, which caused profound and irreversible RPE and cone photoreceptors dysfunction.
Examinations that were performed after 1st, 2nd, 3rd, 4th and 6th months of the treatment consisted of: BCDVA, mfERG and OCT tests (5th month examination failed due to the absence of the patient). The tenets of the Declaration of Helsinki were followed. Informed consent was obtained after the details of the procedures had been fully explained.

BCDVA increased gradually during the treatment from 0.26 to $0.22 \log$ MAR at the 1 st, to $0.14 \log$ MAR at the 2 nd and to $0.1 \log$ MAR at the 3rd follow-up examination (Fig. 4). BCDVA in the right eye remained stable during the next 3 months of treatment. Except the 3rd follow-up examination, central retinal function, presented by P1 wave response density in mfERG improved and recover to normal value during 6 months follow-up. One of the possible explanations of this was unsystematic use of the drops in the 3rd month, which was confirmed by the patient. P1 wave implicit time during first 2 months of treatment shortened, but then it returned to the value before the treatment (Fig. 4).

The improvement of BCDVA in the right eye corresponded with foveal thickness reduction from $482 \mu \mathrm{m}$ initially to $359 \mu \mathrm{m}$ at the $1 \mathrm{st}$ and to $226 \mu \mathrm{m}$ at the 2nd follow-up visit (Fig. 2). At this point, intraretinal 
Fig. 3 Full-field ERG of the ESCS patient

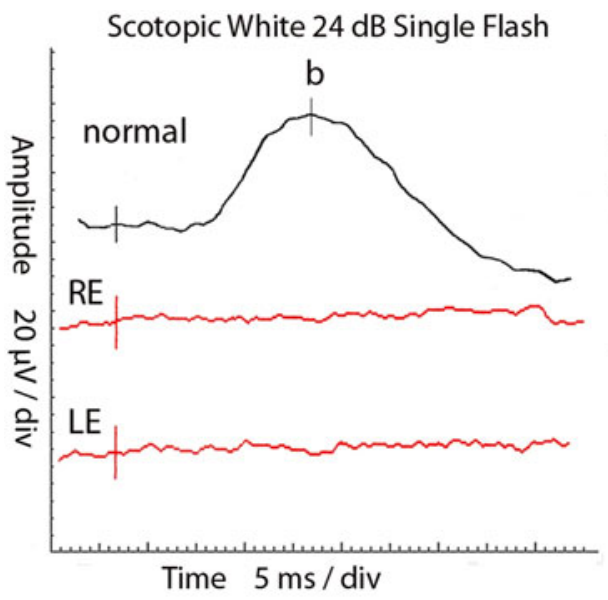

Scotopic White $0 \mathrm{~dB}$ Single Flash

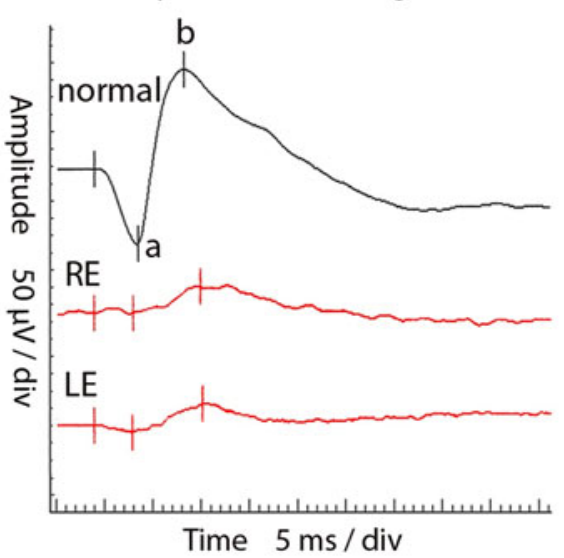

Photopic White $0 \mathrm{~dB}$ Single Flash

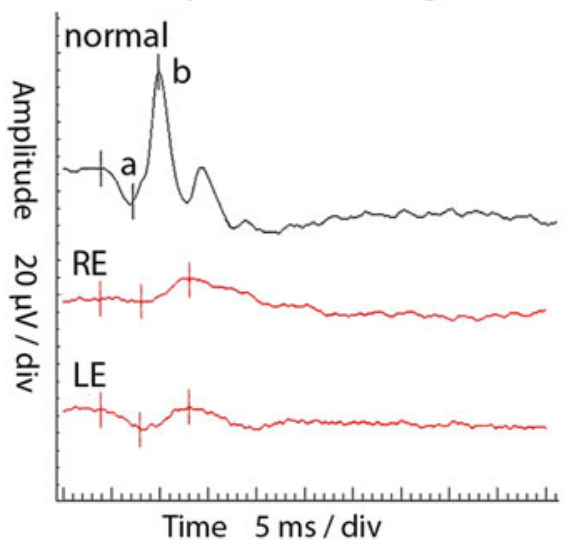

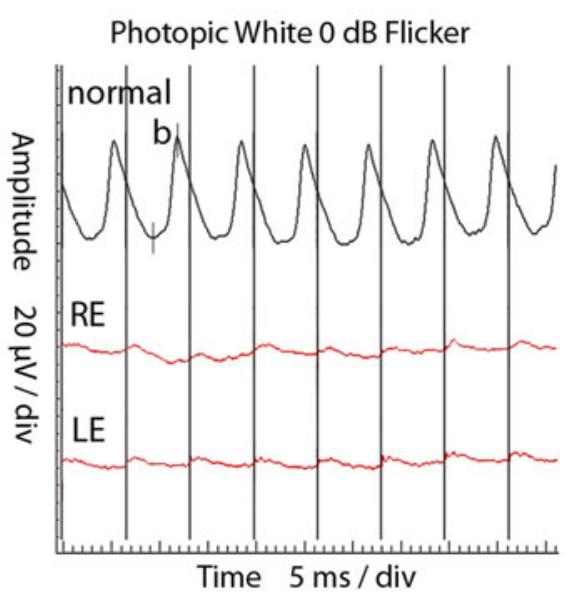

cysts were completely absorbed. Foveal thickness remained unchanged for the next 3 follow-up visits.

\section{Discussion}

The main reason behind the decreased visual acuity in ESCS patients is cystoid changes in macular region. Macular cysts early diagnosis may be of great value for ESCS patients, when at least partial RPE function is preserved. It is possible that by using topical dorzolamide, which provides an increased RPE pumping function and in consequence intraretinal fluid absorption, restoration of normal foveal morphology can be obtained and even in some cases, visual acuity may improve [9-11].

An extracellular membrane-bound carbonic anhydrase (CA XIV) was shown to be present in brain and retina (astrocytes, Muller cells and RPE). Carbonic anhydrase XIV is the target of carbonic anhydrase inhibitors that enhance subretinal fluid absorption in macular edema [14]. Carbonic anhydrase inhibitors usefulness was proven in the treatment of macular cystoid changes associated with diseases such as retinitis pigmentosa [15-18], choroideremia [19] and $\mathrm{X}$-linked retinoschisis [20].

To our best knowledge, this is the third case report study describing topical dorzolamide efficacy in macular cysts management in the ESCS patient, with the longest follow-up period equal to 6 months. Dorzolamide treatment was applied, because we assumed that cystoid macular spaces were filled with endogenous interstitial retinal fluid undetectable to fluorescein angiography testing. Obtained results are in agreement with previously published data [9-11], which presented the visual acuity improvement and foveal structure normalization after dorzolamide treatment.

For the 1st time, mfERG was used to evaluate foveal function during the topical dorzolamide 
Fig. 4 Right eye foveal thickness (FT) and visual acuity (VA) (above); implicit time (ms) and P1wave response density $(\mathrm{nV} /$ $\mathrm{deg}^{2}$ ) of mfERG results (below) of ESCS patient, treated with dorzolamide during 6 consecutive visits
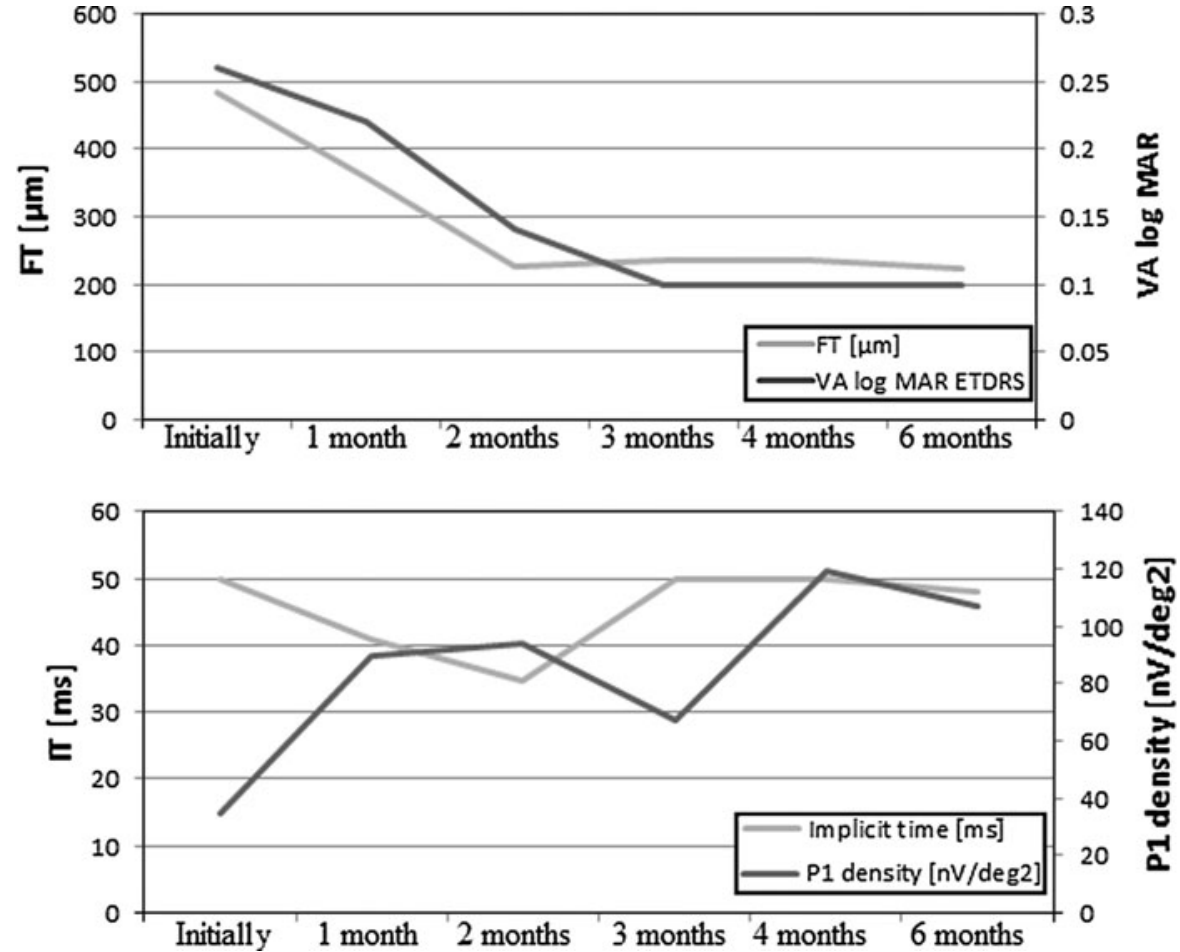

treatment of macular cysts in ESCS. MfERG is a wellknown technique reflecting functional mapping of the retina including foveal region, especially bipolars and cone photoreceptors [21]. To date, to estimate the macular cysts treatment efficacy of ESCS patients visual acuity, retinal thickness measurements (OCT), contrast sensitivity and microperimetry were used [911]. However, visual acuity examination is not an accurate function test. The results of visual acuity measurements are subjective and allow only to estimate retinal function of about 1 central angle degree. Hence, visual acuity examination is not precise enough to estimate retinal function from retinal regions covering macular cysts, which usually surpasses 1 angle degree. That is why we decided to evaluate the retinal function with mfERG. Results of mfERGs provided in this case useful information that visual acuity and foveal thickness improvement corresponded with partial increase in foveal function. Assessment of foveal region function with mfERG was previously described in patients with epiretinal membrane, retinitis pigmentosa, diabetic macular edema, uveitic macular edema and others [22-24].

The results of our study suggest that the use of topical form of carbonic anhydrase inhibitor should be consider for a treatment of macular cysts associated with ESCS.

\section{Conflict of interest None.}

Open Access This article is distributed under the terms of the Creative Commons Attribution License which permits any use, distribution, and reproduction in any medium, provided the original author(s) and the source are credited.

\section{References}

1. Marmor MF, Jacobson SG, Foerster MH, Kellner U, Weleber RG (1990) Diagnostic clinical findings of a new syndrome with night blindness, maculopathy, and enhanced $\mathrm{S}$ cone sensitivity. Am J Ophthalmol 110(2):124-134

2. Haider NB, Jacobson SG, Cideciyan AV, Swiderski R, Streb LM, Searby C, Beck G, Hockey R, Hanna DB, Gorman S, Duhl D, Carmi R, Bennett J, Weleber RG, Fishman GA, Wright AF, Stone EM, Sheffield VC (2000) Mutation of a nuclear receptor gene, NR2E3, causes enhanced $S$ cone syndrome, a disorder of retinal cell fate. Nat Genet 24(2):127-131

3. Audo I, Michaelides M, Robson AG, Hawlina M, Vaclavik V, Sandbach JM, Neveu MM, Hogg CR, Hunt DM, Moore AT, Bird AC, Webster AR, Holder GE (2008) Phenotypic variation in enhanced $\mathrm{S}$-cone syndrome. Invest Ophthalmol Vis Sci 49(5):2082-2093 
4. Sustar M, Hawlina M, Brecelj J (2011) Electroretinographic evaluation of the retinal S-cone system. Doc Ophthalmol 123(3):199-210

5. Jacobson SG, Marmor MF, Kemp CM, Knighton RW (1990) SWS (blue) cone hypersensitivity in a newly identified retinal degeneration. Invest Ophthalmol Vis Sci 31(5):827-838

6. Ganesh A, Stroh E, Manayath GJ, Al-Zuhaibi S, Levin AV (2011) Macular cysts in retinal dystrophy. Curr Opin Ophthalmol 22(5):332-339

7. Wolfensberger TJ (1999) The role of carbonic anhydrase inhibitors in the management of macular edema. Doc Ophthalmol 97(3-4):387-397

8. Marmor MF, Maack T (1982) Enhancement of retinal adhesion and subretinal fluid resorption by acetazolamide. Invest Ophthalmol Vis Sci 23(1):121-124

9. Genead MA, Fishman GA, McAnany JJ (2010) Efficacy of topical dorzolamide for treatment of cystic macular lesions in a patient with enhanced S-cone syndrome. Doc Ophthalmol 121(3):231-240

10. Hajali M, Fishman GA (2009) Dorzolamide use in the management of macular cysts in a patient with enhanced S-cone syndrome. Retin Cases Brief Rep 3(2):121-124

11. Iannaccone A, Fung KH, Eyestone ME, Stone EM (2009) Treatment of adult-onset acute macular retinoschisis in enhanced s-cone syndrome with oral acetazolamide. Am J Ophthalmol 147(2):307-312

12. Marmor MF, Fulton AB, Holder GE, Miyake Y, Brigell M, Bach M (2009) Standard for clinical electroretinography (2008 update). Doc Ophthalmol 118(1):69-77

13. Hood DC, Bach M, Brigell M, Keating D, Kondo M, Lyons JS (2012) ISCEV standard for clinical multifocal electroretinography (mfERG) (2011 edition). Doc Ophthalmol 124(1):1-13

14. Nagelhus EA, Mathiisen TM, Bateman AC, Haug FM, Ottersen OP, Grubb JH, Waheed A, Sly WS (2005) Carbonic anhydrase XIV is enriched in specific membrane domains of retinal pigment epithelium, muller cells, and astrocytes. Proc Natl Acad Sci USA 102(22):8030-8035

15. Ikeda Y, Hisatomi T, Yoshida N, Notomi S, Murakami Y, Enaida H, Ishibashi T (2012) The clinical efficacy of a topical dorzolamide in the management of cystoid macular edema in patients with retinitis pigmentosa. Graefes Arch Clin Exp Ophthalmol 250(6):809-814

16. Apushkin MA, Fishman GA, Grover S, Janowicz MJ (2007) Rebound of cystoid macular edema with continued use of acetazolamide in patients with retinitis pigmentosa. Retina 27(8):1112-1118

17. Fishman GA, Apushkin MA (2007) Continued use of dorzolamide for the treatment of cystoid macular oedema in patients with retinitis pigmentosa. $\mathrm{Br} \mathrm{J}$ Ophthalmol 91(6):743-745

18. Grover S, Apushkin MA, Fishman GA (2006) Topical dorzolamide for the treatment of cystoid macular edema in patients with retinitis pigmentosa. Am J Ophthalmol 141(5):850-858

19. Genead MA, McAnany JJ, Fishman GA (2012) Topical dorzolamide for treatment of cystoid macular edema in patients with choroideremia. Retina 32(4):826-833

20. Khandhadia S, Trump D, Menon G, Lotery AJ (2011) $\mathrm{X}$-linked retinoschisis maculopathy treated with topical dorzolamide, and relationship to genotype. Eye (Lond) 25(7):922-928

21. Hood DC, Frishman LJ, Saszik S, Viswanathan S (2002) Retinal origins of the primate multifocal ERG: implications for the human response. Invest Ophthalmol Vis Sci 43:1673-1685

22. Hwang JU, Sohn J, Moon BG, Joe SG, Lee JY, Kim JG, Yoon YH (2012) Assessment of macular function for idiopathic epiretinal membranes classified by spectral-domain optical coherence tomography. Invest Ophthalmol Vis Sci 53(7):3562-3569

23. Wen Y, Klein M, Hood DC, Birch DG (2012) Relationships among multifocal electroretinogram amplitude, visual field sensitivity, and SD-OCT receptor layer thicknesses in patients with retinitis pigmentosa. Invest Ophthalmol Vis Sci 53(2):833-840

24. Georgiadou E, Moschos MM, Margetis I, Chalkiadakis J, Markomichelakis NN (2012) Structural and functional outcomes after treatment of uveitic macular oedema: an optical coherence tomography and multifocal electroretinogram study. Clin Exp Optom 95(1):89-93 